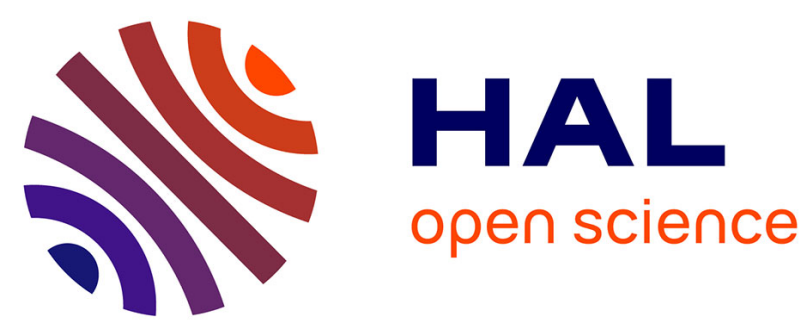

\title{
Focal Hole Shifting of Azimuthally Polarized Sinh Gaussian Beam using Cosine Phase Filter
}

\author{
K Prabakaran, V Karthik, K B Rajesh, P M Anbarasan, V Aroulmoji, A
} Mohamed Musthafa

\section{- To cite this version:}

K Prabakaran, V Karthik, K B Rajesh, P M Anbarasan, V Aroulmoji, et al.. Focal Hole Shifting of Azimuthally Polarized Sinh Gaussian Beam using Cosine Phase Filter. International journal of advanced Science and Engineering, 2020, 06, pp.1476 - 1481. 10.29294/ijase.6.4.2020.1476-1481. hal-03093070

\section{HAL Id: hal-03093070 \\ https://hal.science/hal-03093070}

Submitted on 7 Jan 2021

HAL is a multi-disciplinary open access archive for the deposit and dissemination of scientific research documents, whether they are published or not. The documents may come from teaching and research institutions in France or abroad, or from public or private research centers.
L'archive ouverte pluridisciplinaire HAL, est destinée au dépôt et à la diffusion de documents scientifiques de niveau recherche, publiés ou non, émanant des établissements d'enseignement et de recherche français ou étrangers, des laboratoires publics ou privés. 


\title{
Focal Hole Shifting of Azimuthally Polarized Sinh Gaussian Beam using Cosine Phase Filter
}

\author{
K.Prabakaran ${ }^{1 *}$, V.Karthik ${ }^{1}$, K.B.Rajesh ${ }^{2}$, P.M.Anbarasan ${ }^{3}$, V.Aroulmoji ${ }^{4}$, \\ A.Mohamed Musthafa ${ }^{5}$ \\ ${ }^{1}$ Department of Physics, Mahendra Arts and Science College (Autonomous), Namakkal, Tamilnadu, India \\ ${ }^{2}$ Department of Physics, Chikkanna Government Arts College, Trippur, Tamilnadu, India \\ ${ }^{3}$ Department of Physics, Periyar University, Salem, Tamilnadu, India \\ ${ }^{4}$ Center for Research and Development, Mahendra Engineering College (Autonomous), \\ Namakkal,637503, Tamil Nadu, India \\ ${ }^{5}$ Department of General Studies (Physics Group), Jubail University College (Male Branch) \\ Royal Commission of Jubail, Kingdom of Saudi Arabia
}

\begin{abstract}
Optical intensity distributions in the focal region play an important role in many optical systems. In this paper, the tunable focusing properties of azimuthally polarized sinh Gaussian beams induced by cosine phase masks are investigated theoretically by vector diffraction theory. Results show that when the azimuthally polarized sinh Gaussian beam with cosine phase plate is focused, the focal pattern differs considerably with frequency parameter $(\mathrm{C})$ in the cosine function term. Increasing the value of frequency parameter in the cosine part of the phase mask, focal shift may occur, including multiple-peak focal pattern were also given to show that the focusing properties of this kind of sinh beams may be used to construct tunable optical traps.
\end{abstract}

KEYWORDS: Azimuthally polarized beam,Vector diffraction theory, Sinh Gaussian beams, High NA lens, Optical Trapping.

\section{INTRODUCTION}

Radially and azimuthally polarized vector beams are now among the more well-known cylindrical vector beam categories. Cylindrical vector beams demonstrating cylindrically symmetric polarization have attracted considerable interest because of their potential applications in electron acceleration [1], optical trapping and manipulating [2], and material processing [3]. high resolution microscopy [4] surface plasmon excitation[5], atomic spin analysis [6], and spectroscopy [7]. Two procedures, known as the passive and active methods, generate radially and azimuthally polarized beams [8]. Among these applications, particular interest has been given to the high numerical aperture (NA) focusing property of these beams and their application as a high-resolution probe. Due to the symmetry of the polarization, the electric field at the focus of a cylindrical vector beam has unique polarization properties. For example, it has been shown that the longitudinal component of the focus from such a cylindrical beam is much stronger than the transversal component, and the size of the longitudinal focus is much smaller than the transversal focus $[9,10]$. Recently, one kind of Hermite-sinusoidal-
Gaussian beams, which named sinh-Gaussian beam, has been received intensive attentions [11]. The similarity of hollow sinh-Gaussian (HsG) beam and higher-order Laguerre-Gaussian beam is the intensity null at center. Therefore, a radially polarized HsG beam will show similar focusing characteristics comparing with higher order Laguerre-Gaussian beam. The more general solution is Hermite-Sinusoidal-Gaussian beams, which was introduced by Casperson and coworkers $[12,13]$. Hyperbolic-cosine- Gaussian beams are regarded as the special case of Hermite-sinusoidal-Gaussian beams, and are of practical interest because their profiles can be altered by choosing suitable beam parameters in cosh parts [14].Among the variety of types of gaussian beams, e.g.,Laguerre- Gaussian (LG) beam [15], the high order hollow sinh-Gaussian beam [16], Multigaussian beam [17], Hollow gaussian beam are discussed in depth [18].On the other hand, research on optical vortices has grown rapidly, because optical vortices have some interesting properties and applications [19-20]. Evolution of the optical vortex was found in stochastic vortex fields [21]. Vortex array laser beams have attracted much interest due to their special mesh field distributions, which show great

*Corresponding Author: prabakaran27mar@gmail.com

Received: 15.02.2020 Accepted: 21.03.2020 Published on: 27.03.2020

Prabakaran et al., 
potential in the application of multiple optical traps and dark optical traps [22]. In the investigation of focusing properties of optical beams, tracing the movement of the point of absolute maximum intensity along an optical axis has attracted many researchers for several decades [23-26]. It was found that the point of absolute maximum intensity does not coincide with the geometrical focus but shifts along the optical axis. This phenomenon is referred to as focal shift. More interesting, the focal shift may be in continuous in certain optical focusing systems. To inspect focusing properties of this kind of light beam with azimuthally polarized distribution is very interesting, which may deepen understanding of its properties and expand application. In this paper, the focal hole shift of the azimuthally polarized sinh Gaussian beam with radial cosine phase plate is investigated theoretically by vector diffraction theory.

2. Vector diffraction theory of azimuthally polarized sinh Gaussian beam

A schematic diagram of the suggested method is shown in Figure 1. The azimuthally polarized sinh Gaussian beam with cosine phase plate and then focused through a high NA lens system. The analysis was performed on the basis of Richards and Wolf's vectorial diffraction method [27] widely used for highNA lens system at arbitrary incident polarization. In the case of the incident polarization, adopting the cylindrical coordinates $r, z, \phi$ and the notations of Ref. [28], The focal field of a azimuthally polarized sinh Gaussian beam (APSHGB) can be written as:

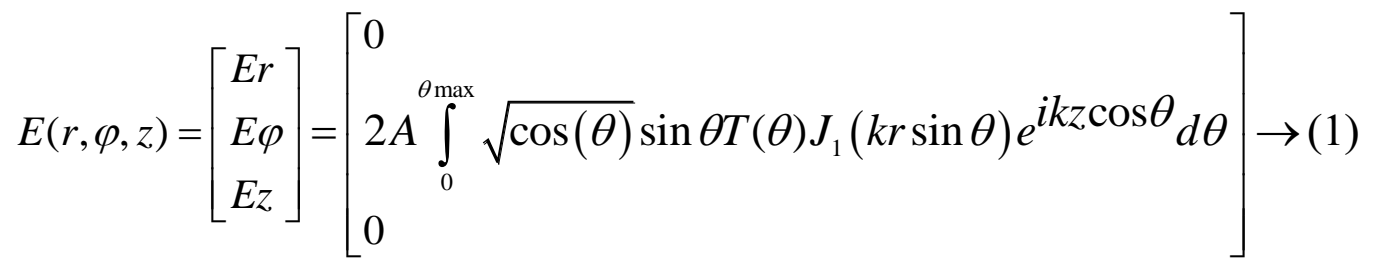

Where, $k=2 \pi / \lambda$ is the wave number and $\mathrm{J}_{\mathrm{n}}(\mathrm{x})$ is the Bessel function of the first kind with order $\mathrm{n}, \mathrm{r}$ and $\mathrm{z}$ are the radial and $\mathrm{z}$ coordinates of observation point in focal region, respectively. In the system investigated in this article, the wave front phase distribution is radial cosine function distribution, and can be written as [29]

$$
\phi=\pi \cdot \cos \left[\pi \cdot \mathrm{C} \frac{\tan (\theta)}{\tan (\theta \max )}\right] \rightarrow(2)
$$

where $\mathrm{C}$ is the frequency parameter in cosine part of the wavefront phase distribution, $\mathrm{C}$ denotes the radial change frequency of the phase and parameter. The reason for choosing this kind of radial cosine phase wavefront is that it is very simple and easy to carry out, for example this kind of phase distribution can be implemented by phase spatial light modulator or by pure phase plate manufactured by the lithographic method conveniently. The $T(\theta)$ describes the azimuthally polarized sinh-Gaussian beam [30], this function is given by

$$
T(\theta)=\sinh ^{m}\left(\frac{\sin (\theta)}{\omega_{0}}\right) \cdot \exp \left(-\frac{\sin ^{2}(\theta)}{\omega_{0}^{2}}\right) \rightarrow(3)
$$

Where $(m=0,1,2, \cdots)$ is the order of the hollow sinh-Gaussian beam. If $m=0$, the above equation is reduced to the fundamental Gaussian beam with beam waist of wo. However, a new kind of sinh Gaussian beam is obtained when $m$ is greater than 1. It is noted that the amplitude distribution of hollow sinh-Gaussian beam is determined by $\omega 0$ and $m$. In order to describe the relation of the amplitude distribution and the parameters of sinh-Gaussian intuitively, the normalized amplitude distribution of sinh-Gaussian with different value of parameter $\omega 0$ and $m$ are shown in Fig. 1 of [30].It is easy to see that the position of maximal amplitude of hollow sinh Gaussian beam is shifted to right while the value of $\omega 0$ or $m$ are increasing. Therefore, one can control the amplitude distribution of hollow sinh-Gaussian beam by choosing $\omega 0$, simultaneously or $m$ reasonably.

\section{RESULTS AND DISCUSSION}

Without loss of validity and generality, the focusing properties of the azimuthally polarized sinh Gaussian beam with radial cosine phase wave front are calculated. We perform the integration of Eq. (1) numerically using parameters $\lambda=1, \theta \max =\arcsin (\mathrm{NA})$, $\omega_{0}=0.500, \mathrm{NA}=0.9$ and $\mathrm{m}=2$. Here, for simplicity, we assume that the refractive index $n=1$ and $A=1$. For all calculation in the length unit is normalized to $\lambda$ and the

\section{Prabakaran et al.,}


energy density is normalized to unity. In order to understand focusing properties of azimuthally polarized sinh gaussian beam with high NA lens system is investigated without wavefront phase modulation. The intensity distribution of aazimuthally polarized sinh gaussian beam incident for the high NA lens is Shown in Fig.2 (a-c).It is observed from the From the Fig.2a, we measured the FWHM of the generated focal hole size is $0.38 \lambda$ and focal depth $4 \lambda$ which is shown in Fig.2b. Such a focal hole segment is useful for low refractive index particle trapping. The Fig.3 (a-f) shows the focal segment generated for frequency parameter values of $C=0.5, C=1$, and $C=1.5$. It can be seen from the
Fig.3 (a\&d) shows that the generated focal segment is a shifted focal hole. From the Fig.3 (a\&d) the maximum intensity position shifted to $-1 \lambda$, However when we increased frequency parameter $(C)$ values for $C=1$ and $\mathrm{C}=1.25$ the maximum intensity position shifted to $-2 \lambda$, and $-2.5 \lambda$ respectively is shown in Fig. 3 (b,e\& c,f). We observed that the generated focal hole in the focal region shifts towards the optical aperture along optical axis on increasing frequency parameter $\mathrm{C}$, namely focal shift phenomenon occurs. If this focal peak is used to construct one optical trap, frequency parameter $\mathrm{C}$ may be employed to adjust trap position, can transport micro particles.

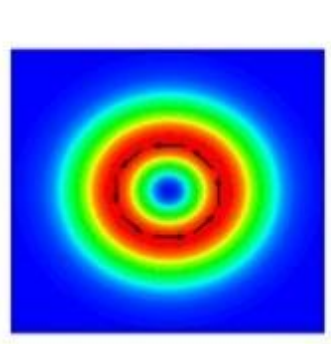

Azimuthally polarized sinh gaussian beam

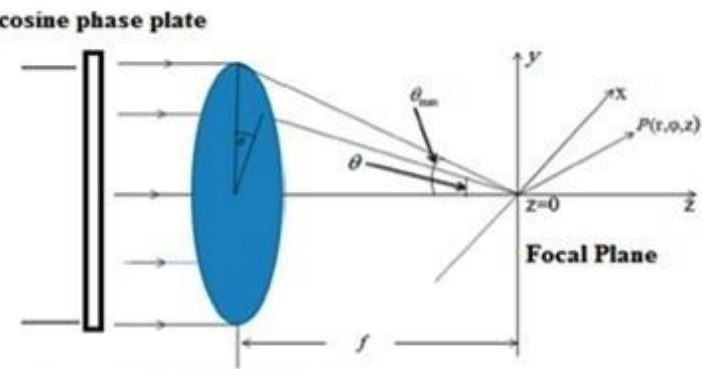

High NA Lens

Fig.1. Focusing of azimuthally polarized sinh gaussian beam with radial cosine phase plate by high NA lens

(a)

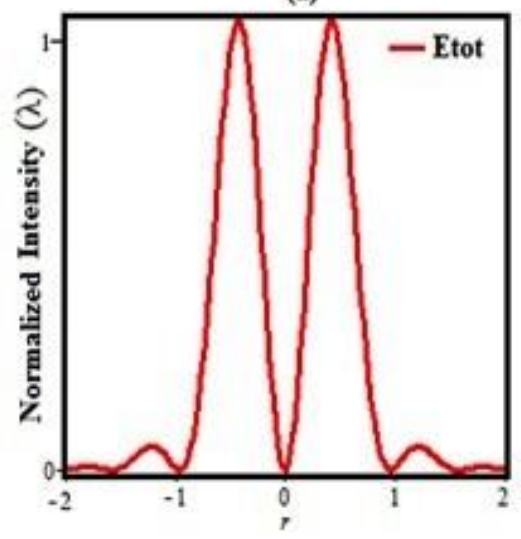

(b)

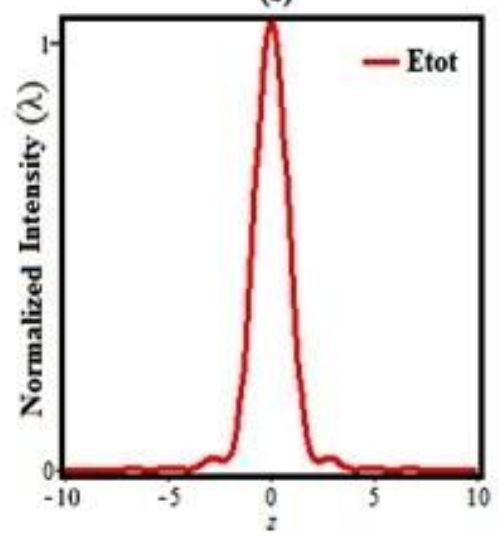

(c)

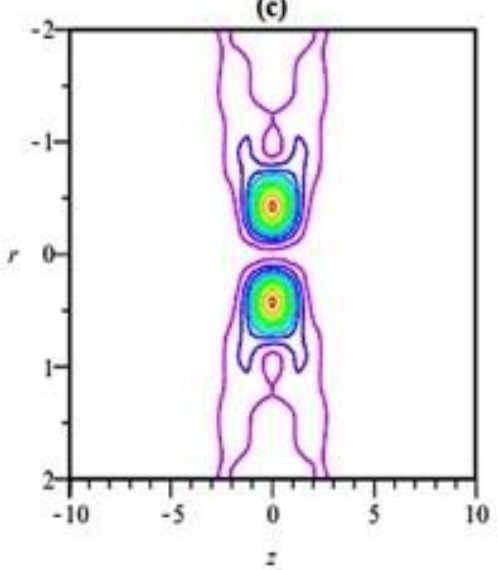

Fig.2.(a) $2 D$ intensity distribution at $\mathrm{z}=0 \lambda$ (b) On axial intensity at $r=0.4 \lambda$ (c) $3 D$ intensity distribution for without radial cosine phase modulation

Prabakaran et al., 

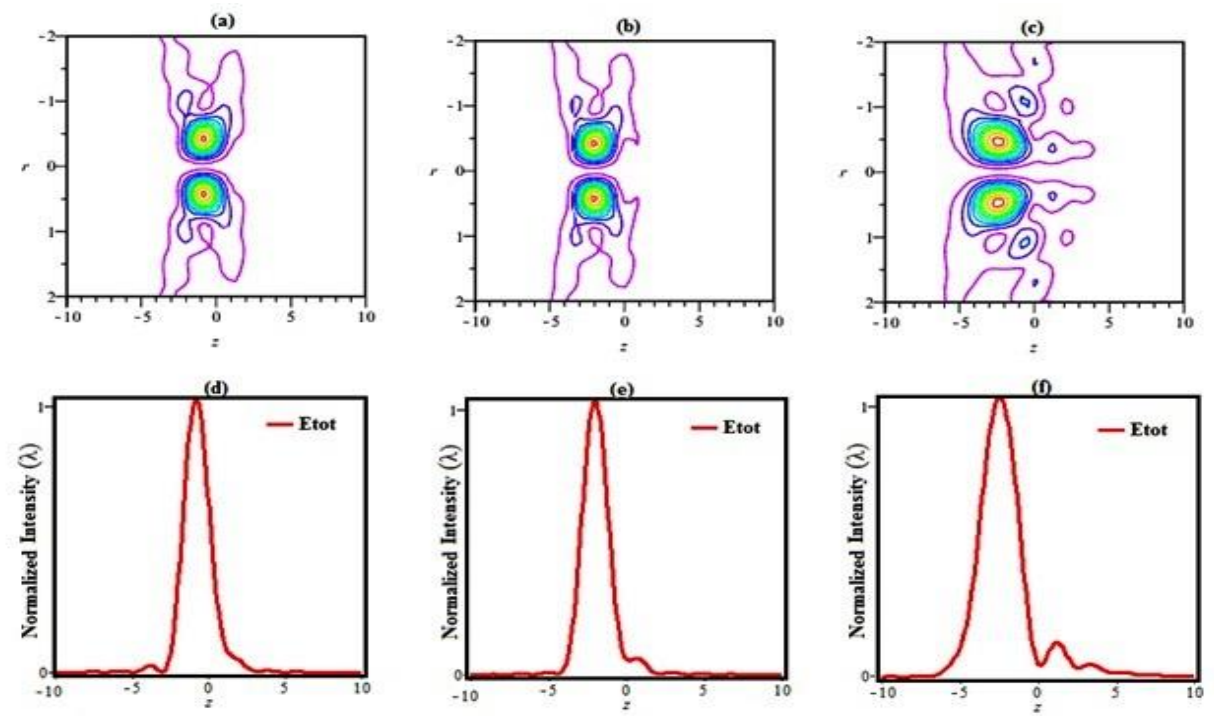

Fig.3. $3 D$ and $2 D$ intensity distribution for ( $a \& d) C=0.5$, (b\&e) $C=1$ and (c\&f) $C=1.5$ respectively.

(a)

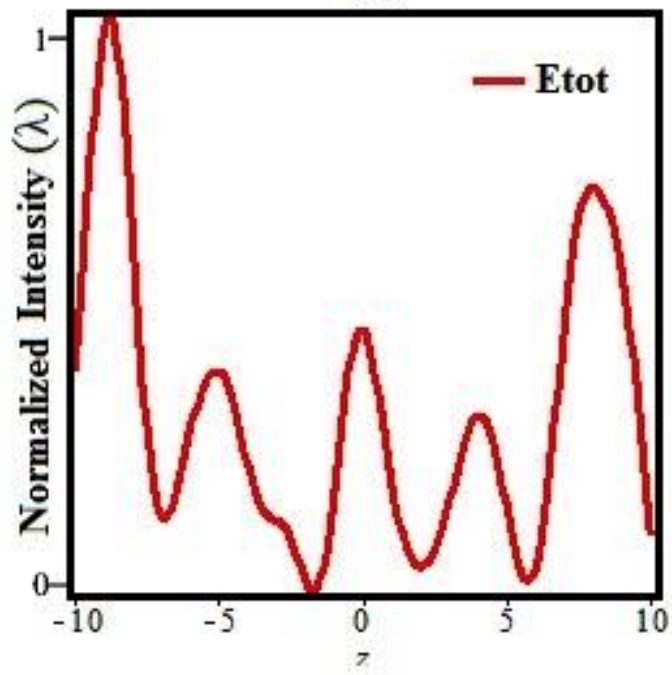

(b)

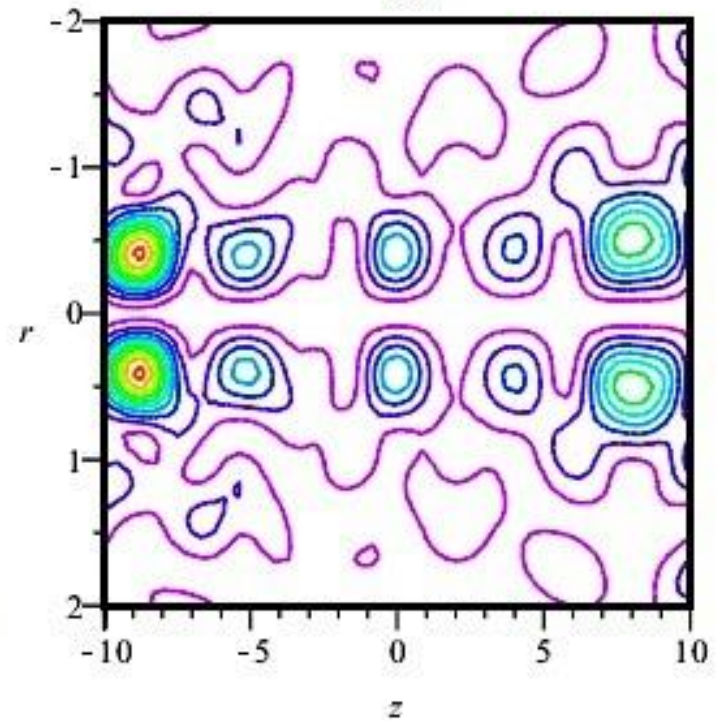

Fig.4.(a) $2 D$ On axial intensity distribution at $r=0.4 \lambda$ (b) $3 D$ intensity distribution for under the condition of $\omega_{0}=0.500, C=6$ respectively.

The Fig.4 shows the focal structure generated by azimuthally polarized sinh gaussian beam tightly focused with high NA lens. It is observed from Fig. $4(a \& b)$ when cosine parameter value $C=6$, the generated focal generated focal structure is a two shifted focal holes along the optical axis, profile having FWHM of $\sim 0.4 \lambda$ and focal depth of $\sim 1.8 \lambda$ which leads to three local intensity minimum occurring between the two intensity peaks. However the maximum intensity positions shifted to $-9 \lambda$, and $+9 \lambda$ respectively is shown in Fig.4. (a\& b). If this focal peak is used to construct multiple optical trap, frequency parameter may be employed to adjust trap position. Now the value of frequency parameters is changed to investigate its effect on focal pattern evolution. The intensity distributions for $\omega_{0}=0.500$ and different $C$ values are illustrated in Fig. 5. It can be seen from this figure that the frequency parameter $\mathrm{C}$ affects focal intensity distribution very considerably. On increasing frequency parameter $\mathrm{C}=8$, it is observed five splitted

\section{Prabakaran et al.,}


focal holes near and far from the optical aperture along optical axis with axially separated by the distance is $5 \lambda$ which is shown in Fig.5(a).However, frequency parameter value further increases to $C=8.5$, five splitted focal hole is changed into three splitted focal holes segment and are axially separated by the distance is $6 \lambda$ respectively is shown in Fig. 5 ( b). From above focal pattern evolution process, we can see that for certain frequency parameter $\mathrm{C}$ can alter considerably intensity distribution in focal region of azimuthally polarized sinh Gaussian beam and many novel focal patterns can occur, which can be used to construct tunable optical traps. Therefore, focusing properties of the radially polarized Sinh Gaussian beam may find very wide application in multiple particle trapping and manipulation
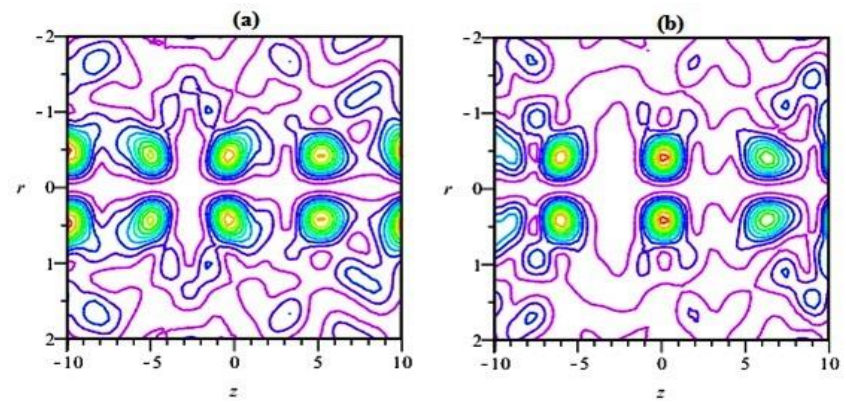

Fig.5. Normalized total intensity distributions in $\mathbf{r - z}$ plane under the condition of $\omega_{0}=0.500$, (a) $C=8$, (b) $\mathrm{C}=8.5$ respectively.

\section{CONCLUSION}

In summary, the focusing properties of a azimuthally polarized sinh gaussian beam by a high NA objective are studied in detail. The simulation results show that the intensity distribution in focal region of the azimuthally polarized sinh gaussian beam can be adjusted considerably by the frequency parameter $\mathrm{C}$. Focal hole can shift along optical axis on increasing $C$, and focal pattern changes are observed. Moreover, by altering frequency parameter of the phase plate will change the energy distributions of maximum intensity peak and other small intensity peaks appeared and novel focal holes also evolve considerably. These special focal fields have potential applications in photolithography, optical trapping and manipulation, nanoscale imaging, and materials processing.

\section{REFERENCES}

[1] Hafizi B, Esarey E, and Sprangle P, 1997.Laserdriven acceleration with Bessel beams, Phys. Rev. E Stat. Phys. Plasmas Fluids Relat. Interdiscip. Topics 55(3), 3539-3545.
[2] Sato S, Harada Y, and Waseda Y, 1994. Optical trapping of microscopic metal particles, Opt. Lett. 19(22), 1807-1809

[3] Niziev V.G. and Nesterov A.V., 1999. Influence of beam polarization on laser cutting efficiency, J. Phys. D Appl.Phys.32(13), 1455-1461.

[4] Failla AV, Jager S, Zuchner T, Steiner M, Meixner AJ. 2007. Topology measurements of metal nanoparticles with $1 \mathrm{~nm}$ accuracy by confocal interference scattering microscopy. Opt Express 15(14), 8532-42.

[5] Lerman GM, Yanai A, Ben-Yosef N, Levy U. 2010. Demonstration of an elliptical plasmonic lens illuminated with radially-like polarized field. Opt Express.18(10), 10871-7.

[6] Fatemi FK. 2011Cylindrical vector beams for rapid polarization-dependent measurements in atomic systems. Opt Express 19(25), 25143-50.

[7] Ivanov D, Shcheslavskiy V, Marki I, Leutenegger M, Lasser T.2009. High volume confinement in two-photon total internal reflection fluorescence correlation spectroscopy. Appl Phys Lett94, 083902.

[8] Zhan Q, 2009. Cylindrical vector beams: from mathematical concepts to applications, Adv. Opt. Photon. 1(1), 1-57.

[9] Quabis S, Dorn R, Eberler M, Glöckl O and G. Leuchs G, 2001 The focus of light- theoretical calculation and experimental tomographic reconstruction, Appl. Phys. B 72, 109-113.

[10] Quabis S, Dorn R, Eberler M, Glöckl O and G. Leuchs G, 2000 Focusing light into a tighter spot, Opt. Commun. 179, 1-7

[11] Sun Q.G, Zhou K.Y, Fang G.Y, Zhang G.Q, Liu Z.J. and Liu S.T, 2012. Hollow sinh-Gaussian beams and theirparaxial properties, Opt. Express 20, 9682-9691.

[12] Casperson L.W., Hall D.G., and Tovar AA, 1997 Sinusoidal-Gaussian beams in complex optical systems, Journal of Optical Society of America A, 14, 3341-3348.

[13] Casperson L.W. and. TovarA.A, 1998.Hermitesinusoidal-Gaussian beams in complex optical systems, Journal of Optical Society of America A, $15,954-961$.

[14] Du. X and Zhao D, 2006 Elliptical cosh-Gaussian beams, Optics Communications, 265, 418-424.

[15] Cagniot Emmanuel, Fromager Michael, Godin Thomas, Passilly Nicolas, AïtAmeur Kamel. 2011.Transverse super resolution technique involving rectified Laguerre-Gaussian LG0p beams. J Opt Soc Am A 28, 1709-1715.

[16] Sun, Q.G., Zhou, K.Y., Fang, G.Y.,Zhang, G.Q., Liu, Z.J.,Liu, S.T. 2012. Hollow sinh-Gaussian beams and their paraxial properties. Opt Express 20, 96829691. 
[17] WilliamCharles,J., Prabakaran,K., Parimaladevi,R., Rajesh,K.B.,. Pillai, T.V.S.2014. Tight focus of aazimuthally polarized and amplitudemodulated annular multi-gaussian beam, Optik 125, 3023-3026.

[18] Prabakaran,K., Rajesh,K.B., Anbarasan,P.M, 2014.Tight focusing of phase modulated radially polarizedhollow Gaussian beam using complex phase filter, Optik 125, 6965-6968.

[19] Mamaev A.V., Saffman M, and Zozulya A. A, 1997.Decay of high order optical vortices in anisotropic nonlinear optical media, Phys. Rev. Lett. 78, 2108-2111.

[20] Trapani P.D, Chinaglia W, Minardi S, Piskarskas A, and Valiulis G, 2000. Observation of quadratic optical vortex solitons, Phys. Rev. Lett. 84, 3843-3846.

[21] Roux F.S, 2011. Evolution of optical vortex distributions in stochastic vortex fields, Proc. SPIE 7950, 79500T

[22] Kuo C.F and Chu S.C, 2013.Calculation of the force acting on a micro-sized particle with optical vortex array laser beam tweezers, Proc. SPIE 8637, 86370A

[23] Li Y, Wolf E, 1981 Focal shifts in diffracted converging spherical waves, Opt. Commun. 39, 211-215.
[24] CorralM.M., Caballero M.T, Escriva L.M, Andres P, 2001 Focal-shift formula in apodized non tele centric focusing systems, Opt. Lett. 26, 1501-1503.

[25] SheppardC.J.R, Totok P, 2003 Focal shift and axial coordinate for high-aperture systems of finite Fresnel number, J. Opt. Soc. Am. A 20, 2156-2162.

[26] Gao X, Zhou F, Xu W, Gan F, 2005 Gradient force pattern, focal shift, and focal switch in an apodized optical system, Optik 116, 99-106.

[27] Richards B, Wolf E, 1959 Electromagnetic diffraction in optical systems, II. Structure of the image field in an aplanatic system. Proc. R. Soc. Lond. A Math. Phys. Sci.253 (1274) 358379

[28] Youngworth K.S, Brown TG, 2000 Focusing of high numerical aperture cylindrical vector beams. Opt. Express 7, 77-87.

[29] Gao X, Li J, Li Z, Wang J, Zhuang S, 2010 Focusing of radially polarized beam with radial cosine phase wavefront, Optik 121, 1674-1680.

[30] JieLin, YuanMa, Peng Jin, Graham Davies, Jiubin Tan, 2013 Longitudinal polarized focusing of radially polarized sinh-Gaussian beam, Opt. Exp, 21, 13193-13198.

All @ 2020 are reserved by International Journal of Advanced Science and Engineering. This Journal is licensed under a Creative Commons Attribution-Non Commercial-ShareAlike 3.0 Unported License.

Prabakaran et al., 